\title{
Leukocytapheresis in patients with inflammatory bowel diseases
}

\author{
Małgorzata Woźniak ${ }^{1}$, Ilona Kurnatowska ${ }^{2,3}$, Ewa Małecka-Panas ${ }^{1}$, Renata Talar-Wojnarowska ${ }^{1}$ \\ ${ }^{1}$ Department of Digestive Tract Diseases, Medical University of Lodz, Lodz, Poland \\ 2Department of Clinical Pharmacology, $1^{\text {st }}$ Chair of Internal Medicine, Medical University of Lodz, Lodz, Poland \\ ${ }^{3}$ Department of Nephrology, Norbert Barlicki University Hospital, Lodz, Poland
}

Gastroenterology Rev 2021; 16 (2): 99-105

DOI: https://doi.org/10.5114/pg.2021.106658

Key words: leukocytapheresis, ulcerative colitis, Crohn's disease.

Address for correspondence: Małgorzata Woźniak, Department of Digestive Tract Diseases, Medical University of Lodz, Lodz, Poland, phone: +48 694279 312, e-mail: mmw89@op.pl

\begin{abstract}
Leukocytapheresis, a blood purification therapy, exerts anti-inflammatory effects by removing activated leukocytes from the peripheral blood through extracorporeal circulation. It is a potential option of treatment for patients with inflammatory bowel diseases (IBD), especially those with ulcerative colitis (UC), who do not respond to conventional therapy. Given that it has a favourable safety profile and seems to have steroid sparing effects, its position in the treatment of UC is likely to expand. However, there is inadequate evidence to draw any conclusions about the efficacy of leukocytapheresis in patients with Crohn's disease, and it should only be used in accordance with special arrangements for consent. Considering the current level of knowledge, it is essential to conduct large, well-designed, randomized clinical trials to evaluate the effects of leukocytapheresis in the management of IBD patients.
\end{abstract}

\section{Introduction}

Inflammatory bowel diseases (IBD) such as Crohn's disease (CD) and ulcerative colitis (UC) are chronic disease of multifactorial aetiology. Our knowledge of the inflammatory mechanism taking part in such diseases has improved in recent years but is still insufficient [1]. Currently available therapy is aimed at nonspecific reduction of inflammation and includes anti-inflammatory and immunosuppressive treatment $[2,3]$. The most effective therapy for patients who do not respond to conventional medications is biological treatment with anti-tumour necrosis factor- $\alpha$ (TNF- $\alpha$ ) antibodies, antiintegrin, or anti-interleukin agents [4], which have changed the approach to the IBD treatment; however, not every patient responds to this treatment, and lack of response is relatively frequent $[5,6]$.

The IBD patients who have active disease in spite of steroids, immunomodulators, or biologic therapy represent a population that is particularly difficult to treat. Other therapeutic methods should be under investigation, and they are eagerly awaited. One potential option of treatment for IBD patients, in particular those with
UC, is leukocytapheresis, a blood purification therapy that exerts anti-inflammatory effects by removing activated leukocytes from the peripheral blood through an extracorporeal circulation. It is known that patients with active IBD have elevated myeloid leucocytes, such as the CD14+CD16+ monocytes, which are a major sources of TNF- $\alpha$ [7]. In selected patients, leukocytapheresis may be an effective complement for conventional treatment and are a considered therapeutic option when immunosuppressive and biologic treatment has no effect or has been discontinued due to adverse effects, and when a patient is not a good candidate for surgical treatment because of a comorbidity.

Leukocytapheresis for treatment of UC patients has been used in some European and Asian countries for several years [8, 9]. In 2000 it was approved by the Japanese Ministry of Health as a treatment option for patients with active UC, and it is recommended for steroid-refractory or steroid-dependent patients with moderate to severe UC. It can be performed a maximum of 10 times under public insurance coverage in Japan [9]. Some studies have suggested that selective apheresis may be of benefit as a steroid-sparing treat- 
ment [10, 11]. Moreover, unlike conventional pharmacological therapy, leukocytapheresis may be associated with a low rate of adverse events. However, most of the published studies are non-controlled, without a placebo arm, and with different leukocytapheresis regimens. Because the cost of this treatment is relatively expensive, further randomized controlled trials are necessary to identify patients who respond well to the apheresis procedure. It might be helpful in selected patients with active UC where satisfactory effects have not been achieved on corticosteroids, or immunosuppressive or biologic therapy.

\section{Leukocytapheresis technique}

Leukocytapheresis aims to reduce the number of white blood cells to dampen the inflammatory reaction. The peripheral blood is passed extracorporeally through a removable column or fibre filter that adsorbs leukocytes while the remaining blood is returned to the patient though a separate intravenous line [12]. Currently, 2 selective adsorption apheresis devices are approved which removed different populations of white blood cells. The Cellsorba system, which contains nonwoven polyester fibres, removes granulocytes, monocytes, lymphocytes, and some platelets. The Adacolumn system contains cellulose diacetate beads that adhere to Fc $\gamma$ receptors on granulocytes and monocytes, and selectively removes them without significantly affecting lymphocytes or platelets. During granulomonocytapheresis (GMA) the column carriers adsorb about $65 \%$ of granulocytes, $55 \%$ of monocytes, and a small fraction of lymphocytes from the blood in the column [13]. In each system, venous blood is removed in a continuous flow, anticoagulated, processed to deplete the leucocytes, and returned to the circulation [14]. Leukocytapheresis is usually repeated 1-3 times per week for 5-8 weeks; the frequency is determined mainly based on the severity of disease and on the patient's tolerance, and the protocol of the procedure is not clearly established $[9,15]$.

The impact of leukocytapheresis on the immune system is potentially very interesting, and the benefits of this treatment are more than just the removal of activated white blood cells. Depletion of granulocytes and monocytes by GMA was associated with reduction of circulating cytokines. These observations support the assumption that peripheral blood granulocytes and monocytes influence the circulating levels of cytokines and should be appropriate targets of the GMA therapy $[13,16]$. The diverted leukocytes are replaced by naïve leukocytes from the bone marrow or peripheral pooling sites. As a result, there is a decrease in leukocyte expression of adhesion molecules and reactive oxygen species. Leukocytapheresis has also been shown to modulate levels of proinflammatory cytokines such as TNF- $\alpha$, interleukins, as IL-1 $\beta$, IL-6, and IL-8, and reduces cell-surface levels of L-selectin, a molecule that plays a key role in initiating leukocyte adhesion to the vascular endothelium [7, 13, 14]. Moreover, adsorbed granulocytes and monocytes release IL-1 receptor antagonist, hepatocyte growth factor, and soluble TNF- $\alpha$ receptors, which are anti-inflammatory factors [7].

\section{Leukocytapheresis efficacy in ulcerative colitis}

The data obtained from published studies are generally quite consistent: apheresis may be a therapeutic option for steroid-dependent UC and may be a useful therapy after the failure of conventional treatments. In the majority of published studies, a remission rate of $39-71 \%$ has been achieved in patients with steroid-dependent or steroid-refractory UC, and the steroid-sparing effects of this treatment have been proven $[8,9$, $15,17,18]$. However, some of the reported data failed to prove the benefits of apheresis treatment $[19,20]$. Moreover, some of the studies concerning leukocytapheresis in UC patients are unblinded, with single-arm design, meaning that statistical comparisons could not be performed.

According to published data, the different types of apheresis were similarly effective in the therapy of patients with moderate to severe UC. In the study of Yamasaki et al. 94 patients were randomly assigned to one of 2 treatments: leukocytapheresis or GMA apheresis. The therapeutic response rate was $82 \%$ for GMA and $70 \%$ for leukocytapheresis without a statistically significant difference between analysed apheresis systems [21].

In a large, prospective, observational study of 623 UC patients treated by leukocytapheresis the clinical remission rates in mild, moderate, and severe UC were $79.5 \%, 67.6 \%$, and $67.2 \%$, respectively, showing no significant difference between the groups. Moreover, there was no difference between the clinical remission rates in the steroid-resistant, steroid-dependent, and nonrefractory patients [15]. In 2016 the results of the ART (Adacolumn in Refractory UC Patients Trial) study were also published. The included patients were steroid-dependent with refractory active UC with insufficient response or intolerance to immunosuppressants and/or biologic agents. After Adacolumn treatment remission was observed in $40.3 \%$ of patients who failed on immunosuppressants and $27.8 \%$ of those who failed on anti-TNF- $\alpha$ treatment. The authors concluded that GMA treatment provided a positive clinical benefit in terms of $U C$ remission in a difficult group of patients with limited effective treatment options [8]. 
A recently published, multicentre study by Yamamoto et al. included patients with Mayo score of $\geq 6$ and active UC despite receiving 1 or more of the medications: 5-aminosalicylic acid, corticosteroids, immunosuppressant, or biologic therapy. The patients with a leukocyte count of $<2000 / \mathrm{mm}^{3}$, serious infection, and bleeding were excluded from the study. Clinical remission after GMA treatment was achieved in $47 \%$ of patients, and improvement was observed in another $25 \%$. The authors reported that patients with a short duration of UC (less than 1 year), with first UC episode, steroid naïve, as well as biologic naïve responded better to GMA [9]. Another interesting study was conducted to determine if the introduction of GMA at an early stage reduces corticosteroid doses and steroid dependency in the 5-year follow-up period. The study included 40 consecutive patients with moderately active symptoms as the first exacerbation of UC. Twenty patients were treated with GMA, with or without corticosteroids (apheresis group), and 20 patients only with corticosteroids (steroid group). During a follow-up period, the mean dose of steroid was significantly lower in the apheresis group than in the steroid group. Furthermore, the incidence of steroid-dependence was significantly lower in the apheresis group at the end of the follow-up period ( $5 \%$ versus $35 \%$; $p<0.05$ ) [10].

The recommended apheresis protocol in active UC has not been established. In a Spanish study the efficacy and safety of different GMA protocols in steroid-dependent UC patients were evaluated. The patients were randomly assigned to receive 5 or 10 GMA sessions over 5 or 10 consecutive weeks, respectively. There was no statistically significant difference in clinical remission achievement; however, patients from group 2 required fewer doses of steroids [22]. However, in the meta-analysis of 9 clinical trials concerning GMA therapy, intensive apheresis ( $\geq 2$ sessions per week) was more effective than weekly apheresis for inducing clinical remission in UC patients [11].

Apheresis may be also a promising therapeutic option in patients with UC and cytomegalovirus (CMV) infection. Co-incidence of those diseases is often refractory to immunosuppressive therapies, and GMA treatment may be helpful in selected patients. Yoshino et al. analysed 11 patients with UC and CMV infection who did not achieve remission after pharmacological UC treatment and antiviral therapy with ganciclovir. Patients were qualified for repeated GMA sessions. Nine of them achieved clinical remission, however in 2 patients colectomy was required because of refractory UC. CMV-DNA was not detected in all 11 patients after GMA. The authors concluded that GMA might be a safe and effective therapeutic option for UC patients positive for CMV, and it may be considered in selected patients [23].
However, the observed mucosal healing rate is usually lower than the clinical remission rate in analysed patients. In the study of Shimoyama et al. only $28 \%$ of UC patients achieved endoscopic remission after GMA treatment, and mucosal healing was more frequently observed in patients with moderate endoscopic activity at entry versus those with severe endoscopic activity. Moreover, the relapse rate was significantly higher in patients who did not achieve mucosal healing during GMA therapy [24].

However, as mentioned above, not all studies in UC patients have shown the effectiveness of apheresis treatment. The largest randomized, double-blind, sham-controlled study of Adacolumn therapy failed to demonstrate efficacy for the induction of clinical remission or response in patients with moderate-to-severe UC. The clinical trial included 168 patients from North American centres and 47 from centres in Europe or Japan. The clinical remission rates were $17 \%$ for the GMA and $11 \%$ for the sham-treatment groups, respectively, and the difference was not statistically significant. Similarly, no changes were observed for the apheresis- and sham-treatment groups for endoscopic remission, and changes in Mayo and quality life scores [19]. The same results were reported by Domenech et al. in analysis of efficacy and safety of adding GMA to oral prednisone in patients with steroid-dependent UC. The authors concluded that the addition of 7 weekly sessions of GMA to a conventional course of oral prednisone did not increase the proportion of steroid-free remissions in patients with active steroid-dependent UC, although it delayed clinical relapse [20].

Moreover, the literature is conflicted regarding the maintenance benefit of apheresis therapy. Some reports are highly suggestive of a prolonged remission or response in a maintenance experience $[11,18,25,26]$, others are unfavourable [27]. lida et al. reported that among patients who responded to GMA, the 3-year sustained clinical remission rates in steroid naïve, steroid-dependent, and steroid-refractory subgroups were $83.3 \%, 68.8 \%$, and $23.1 \%$, respectively. Steroid-naïve patients appeared to benefit the most from the GMA treatment, and attain a favourable long-term clinical course [25]. However, from a clinical point of view, apheresis is not the first line of recommended therapy of UC patients, before corticosteroid treatment. In a recently published study, the long-term outcome of GMA apheresis in steroid-dependent active UC patients with insufficient response to immunosuppressants and/ or TNF inhibitors was assessed. Of 94 patients, steroid-free remission was achieved in $34 \%$ patients at week 24, and in 33\% at week 48 [26].

In the other hand, in an Italian study, all UC patients who responded to apheresis therapy were followed up for 12 months after GMA, and no additional treatment 
was added. At the $9^{\text {th }}$ months of follow-up, 6 of 8 UC patients were still in clinical remission; however, at the $12^{\text {th }}$ month of follow-up, only 1 of 8 UC patients maintained remission [27]. It was also observed that the risk factor for early relapse after apheresis treatment was younger age. The 5-year relapse-free rate in patients aged $>40$ years was $49.9 \%$, which was significantly higher than that in patients aged $\leq 40$ years $(22.9 \%$, $p<0.01$ ) [21]. Considering these results this therapeutic procedure may have potential for treatment in selected UC patients not responding to conventional therapy; however, further randomized studies are necessary.

\section{Leukocytapheresis efficacy in Crohn's disease}

The efficacy of apheresis therapy in CD patients is still controversial and not sufficiently proven, mainly because of a lack of sufficient proper studies [18, 28, 29]. So far, most of research has been focused on analysing leukocytapheresis in patients with UC, which can be caused by differentials in disease locations and connected with this disparity of inflammatory lymphocytes within the inflamed tissues. Lesions located in the colon or in the rectum are strongly affected by neutrophils, while changes in the small intestine in IBD do not have a precisely understood role in myeloid lineage leucocytes [30].

However, several studies have described apheresis as a therapeutic option also in patients with CD. In an Italian, prospective, observational study $35 \mathrm{CD}$ patients who failed to respond or were dependent on steroid therapy were treated with GMA once a week for 5 consecutive weeks. Clinical remission was observed in $63 \%$ of these patients, and at 6 months it was maintained by $54 \%$ of patients. Moreover, after this treatment a relevant proportion of patients (43\%) were still in remission after 12 months [18].

Unfortunately, most of the previously reported studies for GMA in CD examined small numbers patients and were open-label trials. In one such study 7 CD patients who did not respond to conventional therapy were treated by GMA sessions for 5 consecutive weeks. This treatment induced remission in 5 of 7 patients for up to 1 year [28]. In the study of Kusaka et al. 6 patients with active $C D$ unresponsive to conventional medications received GMA treatment. Three of them responded to the therapy, but only in 1 case was remission induced [29]. In another small study analysing GMA treatment in $18 \mathrm{CD}$ patients, $50 \%$ of them achieved clinical remission [31]. Similarly, Fukuda et al. in their multicentre Japanese study, including active CD patients refractory to elemental nutrition therapy, showed significant improvements in CD activity index (CDAI) and quality of life scores after GMA therapy. The leucocyte reduction therapy was effective for inducing remission, with an overall response rate of $52.4 \%$ [32]. It is obvious that these promising data need further randomized, placebo-controlled clinical trials to assess the efficacy of apheresis treatment in CD.

In all of the above-mentioned studies the schemes of GMA treatment were different, and the establishment of an appropriate regimen for this procedure is required. What is more, the time to achieve remission of $C D$ should also be taken into consideration. In one randomized multicentre Japanese study the researchers analysed the efficiency of 2 schemes of GMA procedure. They compared weekly GMA to GMA performed in 2 sessions per week and found that intensive GMA and weekly GMA in those patients have similar remission rates, but the time to remission was significantly shorter and was not connected with increased incidence of side effects in the intensive GMA group [33].

On the other hand, the results of the largest published randomized study did not show a benefit of leukocytapheresis in 157 CD patients. The authors reported that GMA treatment of moderate and severe CD was well tolerated but did not significantly differ in effectiveness over a sham procedure regarding remission or clinical response. Clinical remission was achieved by $17.8 \%$ of patients in the GMA group compared with $19.2 \%$ of those in the sham control group. Clinical response (defined as a $\geq 100$-point decrease in CDAI) was observed in $28 \%$ and $26.9 \%$ of patients, respectively. There were also no changes in quality of life between the analysed groups of patients [34]. Currently, taking into account the results of published studies, leukocytapheresis is not a recommended procedure in CD due to the lack of convincing results of published studies.

\section{Leukocytapheresis in extraintestinal manifestations}

Both CD and UC are associated with a variety of extraintestinal manifestations; some of them, such as pyoderma gangrenosum, may be particularly difficult to treat $[35,36]$. Extraintestinal manifestations of IBD are caused by the same inflammatory processes that cause inflammation in the digestive tract [37]. From all systems affected by inflammation in IBD patients, skin diseases contain large numbers of myeloid leucocytes and T cells [38]. Taking this into account, leukocytapheresis may be an appropriate treatment in selected IBD patients with dermatologic manifestations.

Pyoderma gangrenosum is a skin lesion with necrotic areas of irregular ulceration, present in up to $2 \%$ of IBD patients, particularly in those with UC [39-41]. Until now several cases of pyoderma gangrenosum treated with leukocytapheresis have been reported in IBD 
patients with good results $[42,43]$. One of them was a case of 19-year-old male patient with severe CD complicated with pyoderma gangrenosum with extensive refractory skin lesions located on the legs and buttocks. After 10 sessions of weekly GMA therapy clinical improvements were noticed and skin ulcers and eruptions re-epithelialized [42]. Another published case concerned a female patient hospitalized because of UC exacerbation and rapidly progressive skin lesions, diagnosed as a pyoderma gangrenosum. She was treated with intravenous steroids and GMA in twice-weekly sessions. Positive clinical response in both bowel symptoms and skin lesions was observed even after the $3^{\text {rd }}$ GMA session. At the end of 10 sessions of intensive GMA treatment, skin ulcers become dry and crusted and colonic lesions improvements were confirmed by endoscopy [43].

Until now, pyoderma gangrenosum was treated with leukocytapheresis not only during exacerbation of IBD but also in remission periods or without concomitant systemic disorders $[44,45]$. Japanese researchers presented a case of 60-year-old woman with UC in remission, who suffered for 7 years from skin ulcers due to pyoderma gangrenosum. Because it was refractory to the previous treatment - corticosteroids and immunosuppressants - they chose to use leukocytapheresis once a week. Dramatic improvements were noticed even after the first session, and the lesions cleared up completely 3 months after the $4^{\text {th }}$ apheresis session [44]. A case of another young patient suffering from pyoderma gangrenosum with no systemic disorders was also published. After the $4^{\text {th }}$ session of once weekly GMA treatment, skin lesions from his buttock completely cleared up [45]. The excellent safety profile of GMA has been also confirmed - in none of above cases were any side effects of GMA reported.

In the literature a case of a UC patient with Sweet's syndrome treated with leukocytapheresis was also reported. The patient was admitted to the hospital with haematochezia, diarrhoea, fever, and painful erythematous nodules on the face and arms. Histological examination of skin biopsies confirmed Sweet's syndrome with inflammatory cell infiltration composed mainly of neutrophils without evidence of necrotizing vasculitis. The patient was treated with prednisolone and leukocytapheresis, and the erythematous nodules on the skin, as well as the abdominal symptoms, improved [46].

All of these facts makes leukocytapheresis an attractive option of treatment for patients with dermatological manifestations in which conventional therapy is unsuccessful. However, the lack of randomized prospective trials performed on large populations means that standards for treatment of these patients are difficult to established. There is a need for further data analys- ing the efficacy of leukocytapheresis in extraintestinal manifestations in IBD patients.

\section{Safety of leukocytapheresis}

Leukocytapheresis is well tolerated and safe, even in pregnant women and elderly patients $[47,48]$. Transient adverse events were observed in 10-32\% of patients, and the most frequent were headache, nausea, and fever $[8,9,15]$. These adverse events were not serious in the majority of patients, but about $2 \%$ patients decided to discontinue the treatment [9]. Leukocytapheresis does not incorporate immunosuppression, and adverse events related to infection are very rare, i.e. in $0.4-1.2 \%$ of patients $[8,15]$. In the analysis of Dignass et al. anal abscess was reported in 1 of 86 patients (1.2\%), as well as CMV infection in another patient (1.2\%) [8].

Serious adverse events (SAE) were commonly associated with extracorporeal circulation. The reported SAE included deep vein thrombosis $(0.2 \%)$, hypotension $(0,1 \%)$, anaphylactic shock after anticoagulant $(0.1 \%)$, and infective endocarditis (0.1\%) [15]. Due to the nature of apheresis, vascular access adverse events pose a potential problem for treatment administration; 8-10\% of patients experienced vascular access complications, defined as the inability to cannulate or no flow [8, 15].

The rate of leukocytapheresis adverse events between the non-elderly and elderly UC patients ( $>65$ years) was not different [48]. Moreover, GMA treatment was effective and safe even in pregnant women with active UC, despite other treatment [47]. Takahashi et al. reported the cases of 3 pregnant women who received Adacolumn granulocytapheresis, with up to 10 sessions within 3-6 weeks. Two patients achieved complete remission, and 1 patient clinical remission after additional steroid treatment. There was no SAE, and GMA treatment was assessed as safe and effective in these particularly difficult to treat patients [48].

\section{Problems of leukocytapheresis in IBD}

Most of the published studies are non-controlled, with a heterogeneous IBD population, and different leukocytapheresis regiment. The majority of them are unblinded, with single-arm design, meaning that statistical comparisons could not be performed. Moreover, no formal comparison of various treatment strategies using apheresis has yet been made in order to delineate an optimal treatment scheme. In the other hand, in some of the studies the analysed population was composed of patients who are difficult to treat and for whom the current clinical guidelines are of less use due to a paucity of therapeutic options.

When selecting a treatment option, the cost is an important factor. The cost of leukocytapheresis therapy 
is higher as compared with corticosteroids. However, if leukocytapheresis can spare patients from corticosteroids, and reduce the incidence of steroid-dependency, hospitalization and surgery, in some patients it may be cost-effective [49]. In a comprehensive study, Panés et al. reported that in European Union countries the average annual cost per UC patient treated with conventional medications was very close to the GMA procedure. The authors concluded that incorporating GMA in the therapeutic management of moderate to severe UC patients is cost-effective and implies savings related to the reduction of adverse effects derived from corticosteroid use and to the decreased number of surgical interventions [50]. Nevertheless, since the cost of GMA therapy is relatively expensive, it is important to identify patients who respond well to GMA and avoid futile use of apheresis treatment.

\section{Conclusions}

Given that leukocytapheresis has quite a favourable safety profile, has not been associated with serious longterm adverse events, and seems to have steroid sparing effects, its position in the treatment of UC is likely to expand. Although the evidence level is not striking, the available data suggest that leukocytapheresis may be especially useful in active UC where satisfactory effects have not been achieved from corticosteroids, immunosuppressive or biologic therapy. It may be also an attractive option of treatment for patients with dermatological manifestations in which conventional therapy was not successful. However, there is inadequate evidence to draw any conclusions about the efficacy of leukocytapheresis in CD patients, and it should only be used in accordance with special arrangement for consent. Considering the current level of knowledge, clinicians should remember about this method as an option of management in patients resistant to conventional treatment IBD, but it is essential to conduct large, well-designed, randomised clinical trials to evaluate the effects of leukocytapheresis in the management of IBD patients.

\section{Conflict of interest}

The authors declare no conflict of interest.

\section{References}

1. Kucharzik T, Maaser C, Lügering A, et al. Recent understanding of IBD pathogenesis: Implications for future therapies. Inflamm Bowel Dis 2006; 12: 1068-83.

2. Kozuch PL, Hanauer SB. Treatment of inflammatory bowel disease: a review of medical therapy. World I Gastroenterol 2008; 14: 354-77.

3. Triantafillidis JK, Merikas E, Georgopoulos F. Current and emerging drugs for the treatment of inflammatory bowel disease. Drug Des Devel Ther 2011; 5: 185-210.
4. Danese S, Vuitton L, Peyrin-Biroulet L. Biologic agents for IBD: practical insights. Nat Rev Gastroenterol Hepatol 2015; 12: 537-45.

5. Billioud V, Sandborn WJ, Peyrin-Biroulet L. Loss of response and need for adalimumab dose intensification in Crohn's disease: a systematic review. Am J Gastroenterol 2011; 106: 674-84.

6. Peyrin-Biroulet L, Deltenre P, de Suray N, et al. Efficacy and safety of tumor necrosis factor antagonists in Crohn's disease: meta-analysis of placebo-controlled trials. Clin Gastroenterol Hepatol 2008; 6: 644-53.

7. Hanai H, Takeda $\mathrm{Y}$, Eberhardson $\mathrm{M}$, et al. The mode of actions of the Adacolumn therapeutic leucocytapheresis in patients with inflammatory bowel disease: a concise review. Clin Exp Immunol 2011; 163: 50-8.

8. Dignass A, Akbar A, Hart A, et al. Safety and efficacy of granulocyte/monocyte apheresis in steroid-dependent active ulcerative colitis with insufficient response or intolerance to immunosuppressants and/or biologics [the ART Trial]: 12-week interim results. J Crohns Colitis 2016; 10: 812-20.

9. Yamamoto T, lida T, Ikeya K, et al. A multicenter retrospective study aiming to identify patients who respond well to adsorptive granulomonocytapheresis in moderately to severely active ulcerative colitis. Clin Transl Gastroenterol 2018; 9: 170.

10. Yamamoto T, Umegae S, Matsumoto K. Long-term clinical impact of early introduction of granulocyte and monocyte adsorptive apheresis in new onset, moderately active, extensive ulcerative colitis. J Crohns Colitis 2012; 6: 750-5.

11. Yoshino T, Nakase $\mathrm{H}$, Minami $\mathrm{N}$, et al. Efficacy and safety of granulocyte and monocyte adsorption apheresis for ulcerative colitis: a meta-analysis. Dig Liver Dis 2014; 46: 219-26.

12. Fukunaga K, Matsumoto T. Current status and future perspectives of leukocytapheresis for inflammatory bowel disease. J Gastroenterol Hepatol 2012; 27: 997-1003.

13. Hanai $H$, lida $T$, Yamada $M$, et al. Effects of adacolumn selective leukocytapheresis on plasma cytokines during active disease in patients with active ulcerative colitis. World J Gastroenterol 2006; 12: 3393-9.

14. Katz S. Leukocytapheresis: am "out-of-body" experience in inflammatory bowel disease. Gastroenterol Hepatol 2006; 2: 906-13.

15. Yokoyama Y, Matsuokab K, Kobayashi T, et al. A large-scale, prospective, observational study of leukocytapheresis for ulcerative colitis: treatment outcomes of 847 patients in clinical practice. J Crohns Colitis 2014; 8: 981-91.

16. Saniabadi AR, Tanaka T, Yamamoto T, et al. Granulomonocytapheresis as a cell-dependent treatment option for patients with inflammatory bowel disease: Concepts and clinical features for better therapeutic outcomes. J Clin Apher 2019; 34: 51-60.

17. Lai YM, Yao WY, He Y, et al. Adsorptive granulocyte and monocyte apheresis in the treatment of ulcerative colitis: the first multicenter study in China. Gut Liver 2017; 11: 216-25.

18. Sacco R, Romano A, Mazzoni A, et al. Granulocytapheresis in steroid-dependent and steroid-resistant patients with inflammatory bowel disease: a prospective observational study. J Crohns Colitis 2013; 7: e692-7.

19. Sands BE, Sandborn WJ, Feagan B, et al. A randomized, double-blind, sham-controlled study of granulocyte/monocyte 
apheresis for active ulcerative colitis. Gastroenterology 2008; 135: 400-9.

20. Domènech E, Panés J, Hinojosa J, et al. Addition of granulocyte/monocyte apheresis to oral prednisone for steroid-dependent ulcerative colitis: a randomized multicentre clinical trial. J Crohns Colitis 2018; 12: 687-94.

21. Yamasaki S, Sakata Y, Yoshida H, et al. Shorter relapse-free period after leukocyte removal therapy in younger than older patients with ulcerative colitis. Digestion 2019; 100: 247-53.

22. Ricart E, Esteve M, Andreu M, et al. Evaluation of 5 versus 10 granulocyte aphaeresis sessions in steroid-dependent ulcerative colitis: a pilot, prospective, multicenter, randomized study. World J Gastroenterol 2007; 13: 2193-7.

23. Yoshino T, Nakase $H$, Matsuura $M$, et al. Effect and safety of granulocyte-monocyte adsorption apheresis for patients with ulcerative colitis positive for cytomegalovirus in comparison with immunosuppressants. Digestion 2011; 84: 3-9.

24. Shimoyama T, Yamamoto T, Umegae S, et al. Faecal calprotectin level for assessing endoscopic activity and predicting future clinical course in patients with moderately active ulcerative colitis undergoing granulomonocytapheresis: a prospective cohort study. BMC Gastroenterol 2018; 18: 120.

25. lidaT, Ikeya K, Kato $M$, et al. Adsorptive depletion of myeloid lineage leucocytes as remission induction therapy in patients with ulcerative colitis after failure of first-line medications: results from a three-year real world, clinical practice. Digestion 2017; 96: 119-26.

26. Dignass A, Akbar A, Baumgart DC, et al. Granulocyte/monocyte adsorptive apheresis for the treatment of therapy-refractory chronic active ulcerative colitis. Scand J Gastroenterol 2018 53: 442-8.

27. Giampaolo B, Giuseppe P, Michele B, et al. Treatment of active steroid-refractory inflammatory bowel diseases with granulocytapheresis: our experience with a prospective study. World J Gastroenterol 2006; 12: 2201-4.

28. Matsui T, Nishimura T, Matake H, et al. Granulocytapheresis for Crohn's disease: a report on seven refractory patients. Am J Gastroenterol 2003; 98: 511-2.

29. Kusaka T, Fukunaga K, Ohnishi K, et al. Adsorptive monocyte-granulocytapheresis (M-GCAP) for refractory Crohn's dis ease. J Clin Apheresis 2004; 19: 168-73.

30. Tanaka T, Okanobu H, Yoshimi S, et al. In patients with ulcerative colitis, adsorptive depletion of granulocytes and monocytes impacts mucosal level of neutrophils and clinically is most effective in steroid naïve patients. Dig Liver Dis 2008 40: 731-6.

31. Kosaka T, Sawada K, Ohnisihi K, et al. Effect of leukocytapheresis therapy using a leukocyte removal filter in Crohn's disease. Intern Med 1999; 38: 102-11.

32. Fukuda Y, Matsui T, Suzuki Y, et al. Adsorptive granulocyte and monocyte apheresis for refractory Crohn's disease: an open multicenter prospective study. J Gastroenterol 2004; 39: 1158-64.

33. Yoshimura N, Yokoyama Y, Matsuoka K, et al. An open-label prospective randomized multicenter study of intensive versus weekly granulocyte and monocyte apheresis in active Crohn's disease. BMC Gastroenterology 2015; 15: 163.

34. Sands BE, Katz S, Wolf DC, et al. A randomised, double-blind, sham-controlled study of granulocyte/monocyte apheresis for moderate to severe Crohn's disease. Gut 2013; 62: 128894.

35. Ardizzone S, Puttini PS, Cassinotti A, et al. Extraintestinal manifestations of inflammatory bowel disease. Dig Liver Dis 2008; 40: S253-9.

36. Bernstein CN, Blanchard JF, Rawsthorne P, et al. The prevalence of extraintestinal diseases in inflammatory bowel disease: a population-based study. Am J Gastroenterol 2001; 96: 1116-22.

37. Danese S, Semeraro S, Papa A, et al. Extraintestinal manifestations in inflammatory bowel disease. World J Gastroenterol 2005; 11: 7227-36.

38. Saniabadi AR, Hanai $H$, Suzuki Y, et al. Adacolumn for selective leukocytapheresis as a non-pharmacological treatment for patients with disorders of the immune system: an adjunct or an alternative to drug therapy? J Clin Apher 2005; 20: 171-84.

39. Su WP, Davis MD, Weenig RH, et al. Pyoderma gangrenosum: clinicopathologic correlation and proposed diagnostic criteria. Int J Dermatol 2004; 43: 790-800.

40. Annese V. A review of extraintestinal manifestations and complications of inflammatory bowel disease. Saudi J Med Med Sci 2019; 7: 66-73.

41. Greuter T, Vavricka SR. Extraintestinal manifestations in inflammatory bowel disease - epidemiology, genetics and pathogenesis. Expert Rev Gastroenterol Hepatol 2019; 13: 307-17.

42. Ohmori T, Yamagiwa A, Nakamura I, et al. Treatment of pyoderma gangrenosum associated with Crohn's disease. Am J Gastroenterol 2003; 98: 2101-2.

43. Ohno $M$, Koyama S, Ohara $M$, et al. Pyoderma gangrenosum with ulcerative colitis successfully treated by the combination of granulocyte and monocyte adsorption apheresis and corticosteroids. Intern Med 2016; 55: 25-30.

44. Wantanabe $\mathrm{Y}$, Yamada $\mathrm{H}$. Leukocyte adsorption apheresis for the treatment of pyoderma gangrenosum. J Dermatol 2008; 35: 792-4.

45. Kanekura T, Kawahara K, Maruyama I, et al. Treatment of pyoderma gangrenosum with granulocyte and monocyte adsorption apheresis. Ther Apher Dial 2005; 9: 292-6.

46. Terai T, Sugimoto $M$, Osawa $S$, et al. Successful treatment of ulcerative colitis complicated by Sweet's syndrome by corticosteroid therapy and leukocytapheresis. Clin J Gastroenterol 2011; 4: 151-6.

47. Takahashi $H$, Sugawara K, Sugimura M, et al. Flare up of ulcerative colitis during pregnancy treated by adsorptive granulocyte and monocyte apheresis: therapeutic outcomes in three pregnant patients. Arch Gynecol Obstet 2013; 288: 341-7.

48. Komoto S, Matsuoka K, Kobayashi T, et al. Safety and efficacy of leukocytapheresis in elderly patients with ulcerative colitis: the impact in steroid-free elderly patients. J Gastroenterol Hepatol 2018; 33: 1485-91.

49. Shiraki M, Yamamoto T. Steroid-sparing strategies in the management of ulcerative colitis: efficacy of leukocytapheresis. World J Gastroenterol 2012; 18: 5833-8.

50. Panés J, Guilera M, Ginard D, et al. Treatment cost of ulcerative colitis is apheresis with Adacolumn cost effective? Dig Liver Dis 2007; 39: 617-25.

Received: 22.09 .2019

Accepted: 30.06 .2020 